\title{
Erratum: Observation of the motional Stark shift in low magnetic fields [Phys. Rev. A 96, 043401 (2017)]
}

Manuel Kaiser, Jens Grimmel, Lara Torralbo-Campo, Markus Mack, Florian Karlewski, Florian Jessen,

Nils Schopohl, and József Fortágh

(Received 13 July 2018; published 30 July 2018)

DOI: 10.1103/PhysRevA.98.019904

In our article we have estimated that the difference between $\mathbf{v}^{(A)}$ and $\frac{\hbar \mathbf{k}}{M}$ results in a correction of $\approx 100 \mathrm{~m} / \mathrm{s}$ for rubidium Rydberg levels with $n=150$ in a magnetic field of $B=100 \mathrm{G}$. This estimate was based on an erroneous conversion of the numerically calculated permanent electric dipole moments of the states in this regime. Here we want to give a simplified estimate, assuming that the permanent electric dipole moment of a Rydberg state in the presence of a small (Lorentz) electric field is essentially limited to the radial extent of the wave-function $r \propto n^{2}$. This results in a velocity correction given by

$$
\left|\mathbf{v}_{\text {corr }}^{(A)}\right|=\frac{|e| B r}{2 M} \approx \frac{|e| B n^{2}}{2 M} \times 1 \AA .
$$

Thereby, we obtain a correction of $v_{\text {corr }}^{(A)} \approx 0.012 \mathrm{~m} / \mathrm{s}$ for $M=87 \mathrm{u}, n=150$, and $B=100 \mathrm{G}$, which is unlikely to be observable. One would need unrealistic principal quantum numbers as high as $n=10000$ to achieve even a few $10 \mathrm{~m} / \mathrm{s}$ in the same magnetic field. However, the effect could still be observable for lighter atoms, such as lithium or hydrogen, and at higher magnetic fields. We would like to stress that the erroneous conversion in the estimate does not affect the other results and conclusions in our article. 